\title{
Electrically Assisted Stretch Bending of Aluminum Extruded Profile
}

\author{
Fengchao Cao ${ }^{1,2,3}$, Yuansong Zeng ${ }^{1,2,3}$ and Baosheng Liu ${ }^{1,2,3, a}$ \\ ${ }^{1}$ Metal Forming Department, Beijing Aviation Manufacture Technology Research Institute, 100024 Beijing, China. \\ ${ }^{2}$ Aeronautical Key Laboratory for Plastic Forming Technologies, 100024 Beijing, China. \\ ${ }^{3}$ Beijing Key Laboratory of Digital Plastic Forming Technology and Equipment, 100024 Beijing, China.
}

\begin{abstract}
Aluminum extruded profile is widely applied in aircraft frame parts whose forming quality is directly related to the assembly accuracy, aerodynamic shape and the service life of aircraft. However, it normally faces difficulties of large springback and low forming limit while forming at room temperature by stretch bending method. Here, electricity was applied in stretch bending process to heat aluminum profile aiming at improving the formability and reducing the springback by using electrically assisted stretch bending machine which is consisted of electrical power with the capability of $10000 \mathrm{~A}$ and $40000 \mathrm{kN}$ stretching force for each hydraulic cylinder. In this research, a set of uniaxial tension tests was performed at different temperature to determine the relationship between the deformation behavior of aluminum profile and temperature. Then, a series of stretch bending tests were conducted to investigate the springback law of the aluminum profile. The corresponding stretch bending parts were obtained under different conditions. The experiment results show that, the plasticity of aluminum profile can be improved when the temperature rises, and the springback of aluminum profile can be reduced even more due to the electrical heating effect compared to the cold stretch bending process. Therefore, the forming accuracy of aluminum profile can be improved in electrically assisted stretch bending process.
\end{abstract}

\section{Introduction}

Aluminum extruded profile is frequently used in aerospace applications due to their advantages of low density, high stiffness, excellent corrosion resistance and easy to recycle[1-3].The use of aluminum material can meet the requirements of aerospace industry for lightweight and high-performance, i.e., improving the carrying capacity of aerocraft, increasing the cruise range and reducing fuel consumption. Currently, aluminum alloys are the main material component of subsonic aircraft, and also occupy a certain proportion of application on supersonic aircraft. The dosage of aluminum alloys on civil and Military aircrafts are 70\% $\sim 80 \%$ and $40 \% \sim 60 \%$ respectively.

Among various extrusion bending methods, stretch bending is a feasible and well-suited one for achieving stable bending of the extrusions. Conventional stretch bending process of aluminum extruded profiles is conducted at room temperature. However, the formability of aluminum is generally low in that case. Besides, the stretch bending parts are always accompanied with large springback, which limits their application. Nevertheless, some researchers[4-6]found that the plasticity of aluminum can be enhanced greatly when the temperature is risen up to a certain degree. At 1946, the warm forming performance of aluminum alloy sheet was studied. The results showed that the deep drawing property could be significantly improved in warm forming condition. Later on, the warm formability of aluminum sheet was widely investigated in some European developed countrys, and part of the research achievements already have been applied in automobile industry $[7,8]$.

Despite the importance of aluminum extruded profiles, research on hot stretch bending of aluminum profiles still remains empty. The overall objective of this study is to determine the influence of temperature on the stretch bending quality of aluminum profiles. Since electrically assisted manufacturing has shown the advantage of modifying material properties of a metal simply by applying electricity to the metal during deformation[9], electricity was utilized to heat aluminum profiles for improving the formability and reducing the springback in this work. Firstly, stretch bending model was developed. Secondly, a set of uniaxial tension tests was carried out to determine the relationship between the deformation behavior of aluminum profile and temperature. Then, stretch bending tests were conducted based on the obtained uniaxial tension results. Lastly, the springback of stretch bending parts under different conditions were presented and discussed.

* Corresponding author: herr.liubaosheng@163.com 


\section{Stretch bending analysis}

The analytical model is developed for the stretch bending process. The basic hypotheses are as follows: Any cross-section remains planar throughout deformation; The stress across the thickness of the section and the friction between profile and die are ignored, i.e., the stress state is uniaxial; The strain state is proportional to $\mathrm{Y}$ coordinate as shown in Figure.1(b); Homogeneous, isotropic material behavior is adopted; The Bauschinger effect is taken into account while reverse loading and unloading. The relationship between stress and strain is shown in Eq.(1).

$$
\sigma=\left\{\begin{array}{cl}
\varepsilon E & \varepsilon \leq \varepsilon_{S} \\
\sigma_{S}+D\left(\varepsilon-\varepsilon_{S}\right) & \varepsilon>\varepsilon_{S}
\end{array}\right.
$$

Where $\sigma$ and $\varepsilon$ stand for stress and strain respectively, $E$ indicates the Young's modulus, $\sigma_{S}$ is the yield stress, $D$ is the strain hardening constant. The stretch bending method in this work is conducted in three steps, as follows.

1.Pre-stretching

The profile is pre-stretched with the jaws to yield stress(along path "OSA" in Fig.1(a)), the strain component $\varepsilon_{\text {pre }}$ is described as

$$
\varepsilon_{\text {pre }}=u_{\text {pre }} / L_{0}
$$

Where $u_{\text {pre }}$ is pre-stretching displacement, $L_{0}$ is the original length of extrusion.

\section{Bending}

The jaws move to accomplish an increasing contact between the die and the profile until bending process is complete(along path " $\mathrm{AB}$ " for outer surface, along path "ADE" for inner surface in Fig.1(a)), the strain component $\varepsilon_{b e n d}$ is given by

$$
\varepsilon_{\text {bend }}=\varepsilon_{\text {pre }}+\frac{y-y_{n}}{R_{n}}
$$

Where $y$ is the through-height coordinate, taking values between 0 and $b, R_{n}$ is the radius of the neutral axis.

3. Post-stretching

An additional post-stretch displacement $\Delta \varepsilon$ is applied with the jaws after bending process(along path "BC" for outer surface, along path "EFA" for inner surface in Fig.1(a)), the strain component $\varepsilon_{\text {post }}$ is given by

$$
\varepsilon_{\text {post }}=\Delta \varepsilon+\varepsilon_{\text {bend }}
$$
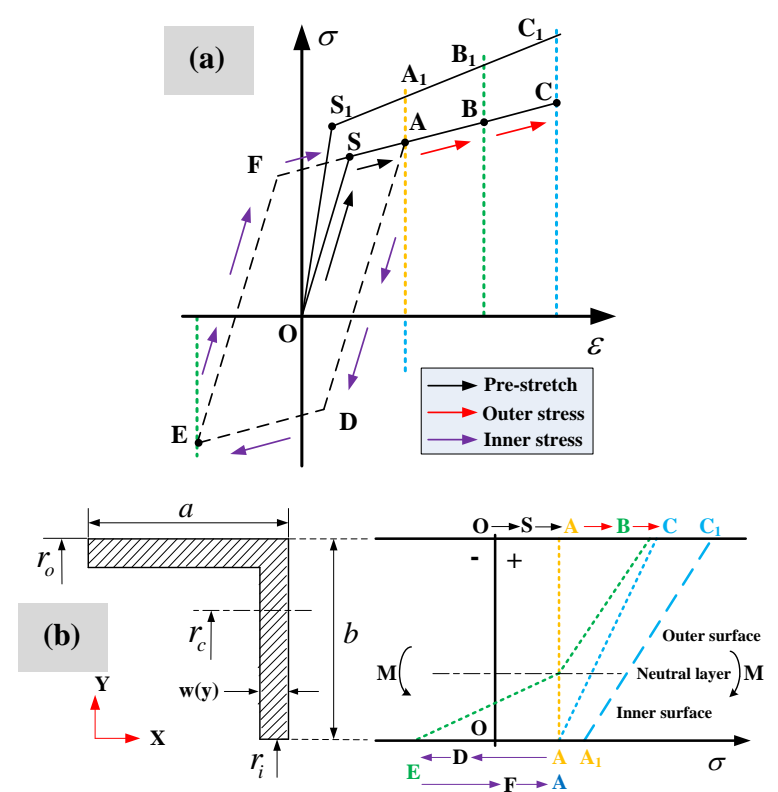

Figure 1. Stretch bending analysis (a)the variation of stress during stretch bending process (b)the stress distribution of "L" cross section

Springback is defined by radius springback, $\Delta R / R$, given by Eq.(5). The moment $(M)$ at the end of the post stretch stage for an extrusion can be evaluated by Eq.(6). The stress $\sigma_{\text {post }}$ can be evaluated using Eq.(1).

$$
\begin{gathered}
\frac{1}{R}-\frac{1}{r}=\frac{M}{E I}, \frac{\Delta R}{R}=\frac{r-R}{R}=\frac{R M}{E I-R M} \\
M=\int_{0}^{b} \sigma_{p o s t}(y) \bullet\left(y-y_{c}\right) \bullet w(y) \bullet d y
\end{gathered}
$$

Where $I$ is the moment of inertia of the cross section, $w$ is the width of the extrusion where the coordinate is $y$, and $b$ is the section height, $y_{c}$ is the coordinate of the centroid of the L-section extrusion before the force is unloaded.

The stress distribution of "L" cross section during stretch bending process is exhibited in Fig. 1(b), which is corresponding to the stretch bending process in Fig.1(a). Comparing with the stress distributions in Fig.1(b) which are based on the two kinds of stress-strain relationship in Fig.1(a), it can be found that the value of stress in the same layer of the profile increases as strain hardening constant $D$ and yield stress $\sigma_{S}$ increase. Therefore, the deformation behaviors of material directly affect the springback of stretch bending parts according to the calculating formula for springback.

\section{Material and experiment}

\subsection{Materials}

The extruded profiles used in the experiment are T5 temper 6063 aluminum extruded profiles with "L" cross section which length and thickness are $1800 \mathrm{~mm}$ and 2.5 $\mathrm{mm}$ respectively. The specimens applied in uniaxial tensile tests were prepared by wire electrode cutting 
from the aluminum extruded profiles, and the tensile direction is in consistent with the longitudinal direction. The detailed information of the uniaxial tensile specimen are exhibited in Fig.2.
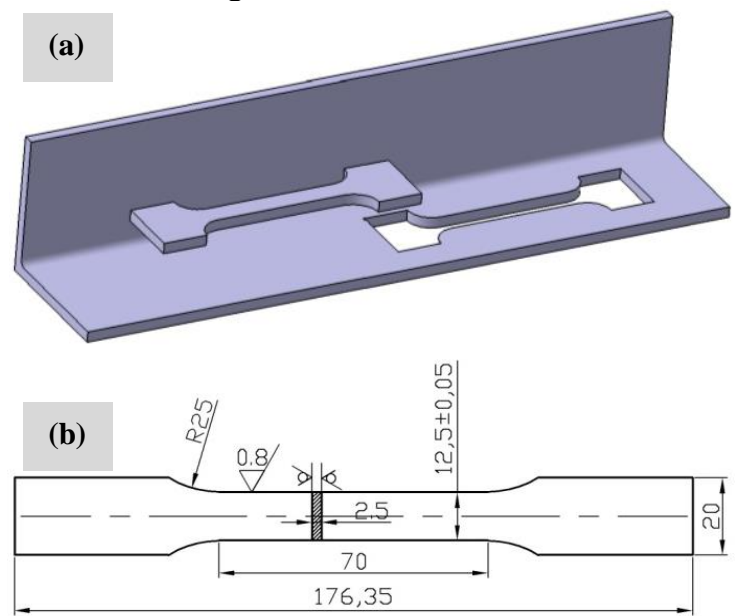

Figure 2. Schematic diagram of tensile specimen (a)3D simulation diagram (b)geometrical shape and size of specimen

\subsection{Uniaxial tensile tests}

The uniaxial tensile tests were carried out on the DDL50 high temperature electric universal testing machine which is equipped with Epsilon3549-25mm Extensometer. The specimens were heated to the test temperature at a rate of $30 \mathrm{~K} / \mathrm{min}$. The tests were performed at temperatures of $293 \mathrm{~K}, 373 \mathrm{~K}, 423 \mathrm{~K}, 473 \mathrm{~K}$, $523 \mathrm{~K}$ and tensile velocities of $1 \mathrm{~mm} / \mathrm{min}$ and $5 \mathrm{~mm} / \mathrm{min}$ according to "GB/T 4338-2006". At last, the true stressstrain curves were obtained from the uniaxial tensile tests.

\subsection{Electrically assisted stretch bending tests}

Electrically assisted stretch bending tests were performed by using electrically assisted stretch bending machine which is consisted of electrical power with the capability of $10000 \mathrm{~A}$ and $40000 \mathrm{kN}$ stretching force for each hydraulic cylinder, as shown in Fig.3(a). A layer $(0.5 \mathrm{~mm})$ of ceramic coating was formed on the surface of $\operatorname{die}(\mathrm{R}=1000)$ to make it insulated, which can keep direct current always only flowing through the profiles during the whole stretch bending process, as shown in Fig.3(b). The parameters of stretch bending tests are shown in TABLE 1.

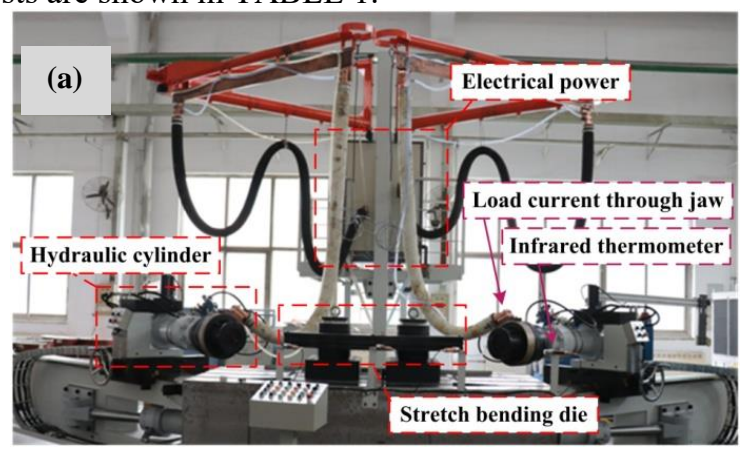

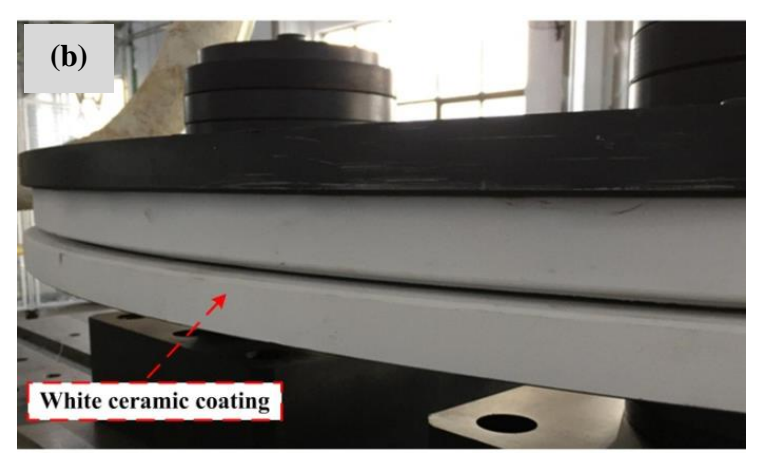

Figure 3. Electrically assisted stretch bending test conditions (a)stretch bending machine (b)stretch bending die

Table 1. The parameters of stretch bending tests

\begin{tabular}{|c|c|c|c|}
\hline Number & $\begin{array}{c}\text { Temperature } \\
/ \mathrm{K}\end{array}$ & $\begin{array}{c}\text { Pre- } \\
\text { stretching } \\
\text { elongation } \\
\text { (yield } \\
\text { strain) } / \%\end{array}$ & $\begin{array}{c}\text { Post- } \\
\text { stretching } \\
\text { elongation } / \%\end{array}$ \\
\hline 1 & 293 & 0.3 & 1.2 \\
\hline 2 & 293 & 0.3 & 1.4 \\
\hline 3 & 293 & 0.3 & 1.6 \\
\hline 4 & 293 & 0.3 & 1.8 \\
\hline 5 & 373 & 1.3 & 1.8 \\
\hline 6 & 423 & 1.3 & 1.8 \\
\hline 7 & 473 & 1.3 & 1.8 \\
\hline 8 & 523 & 1.3 & 1.8 \\
\hline
\end{tabular}

\section{Results and discussion}

The results of uniaxial tensile tests of 6063 aluminum alloy at temperatures of $293 \mathrm{~K}, 373 \mathrm{~K}, 423 \mathrm{~K}, 473 \mathrm{~K}, 523 \mathrm{~K}$ and tensile velocities of $1 \mathrm{~mm} / \mathrm{min}$ and $5 \mathrm{~mm} / \mathrm{min}$ are shown in Fig.4. The true stress-strain curves of uniaxial tensile tests show that, at a given tensile velocity, the flow stress increases with the decreases of temperature. The elongation increases with the increases of temperature within a certain temperature range. The maximum elongation is achieved at $473 \mathrm{~K}$. When the test temperature is raised to $523 \mathrm{~K}$, the elongation becomes lower in comparison with that of $423 \mathrm{~K}$ and $473 \mathrm{~K}$. At the same temperature, the flow stress barely changes as tensile velocity increases, the elongation shows a slight improvement while the tensile velocity decreases from $5 \mathrm{~mm} / \mathrm{min}$ to $1 \mathrm{~mm} / \mathrm{min}$. The strain hardening constant $D$ and yield stress $\sigma_{S}$ decrease with the increases of temperature based on the deformation behaviors below. Given the above results, the plasticity of 6063 aluminum profile can be improved when the temperature rises. 

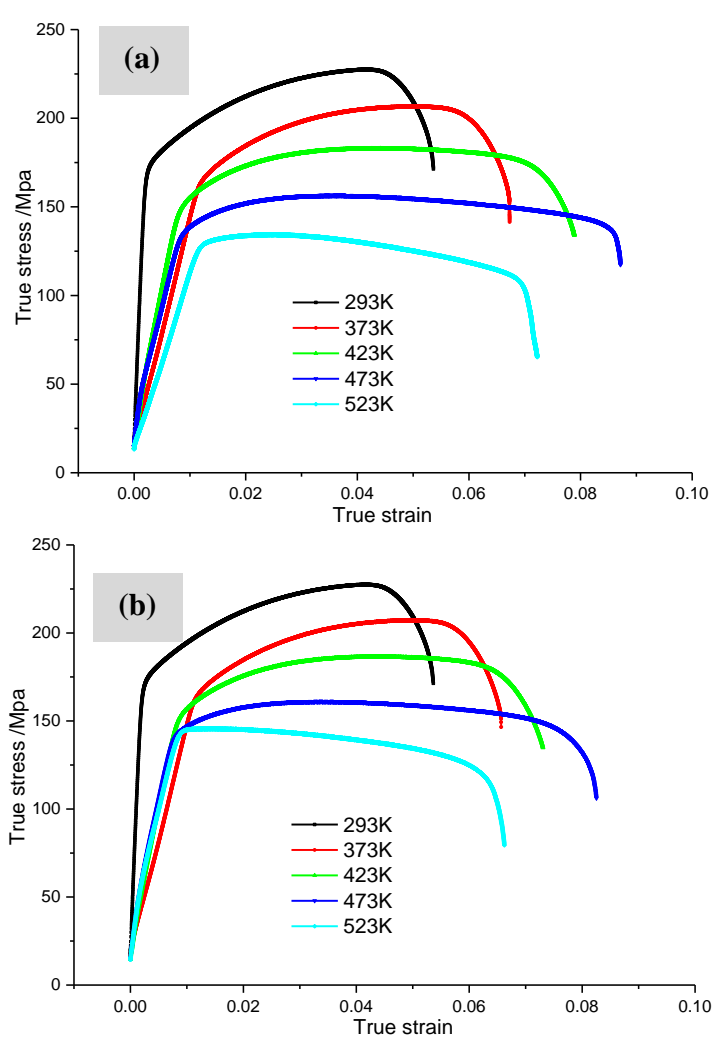

Figure 4. True stress-strain curves of uniaxial tensile tests under different conditions (a) $1 \mathrm{~mm} / \mathrm{min}$ (b) $5 \mathrm{~mm} / \mathrm{min}$

The stretch bending parts of 6063 aluminum profiles under different deformation conditions are shown in Fig.5(a). From Fig.5(b), it is found that the springback of the profiles decreases with the increases of post-stretch elongation at the same temperature. This phenomenon is in agreement with previous research findings. The results of electrically assisted stretch bending tests indicate that the springback of the profiles decreases with the increases of temperature in the condition of same poststretch elongation within a certain range. This can be attributed to the effect of the deformation behaviors of 6063 aluminum at different temperatures. As the temperature increases, lower $\sigma_{S}$ and $D$ can be achieved that will lead to decreased $\sigma_{A}$ and $\sigma_{C}$ as shown in Fig.1(b). So in conclusion the springback is in direct proportion to the moment $(M)$ which decreases with the increases of temperature under the same stretch bending conditions according to the results of the stretch bending analysis and the uniaxial tensile tests obtained above. Comparing with the experimental and analytical springback in Fig.5(b), it is found that the analytical predictions for radius springback are in the consistent tendency as the experimental results. The deviation between them is mainly due to the hypotheses in stretch bending model and inhomogeneous distribution of temperature during electrically assisted stretch bending process. Hence, it can be concluded that the forming accuracy of the 6063 aluminum profile can be improved as the temperature increases.

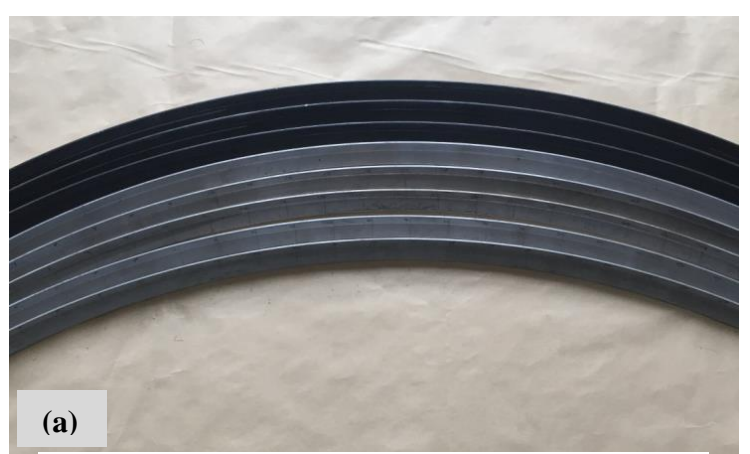

(a)

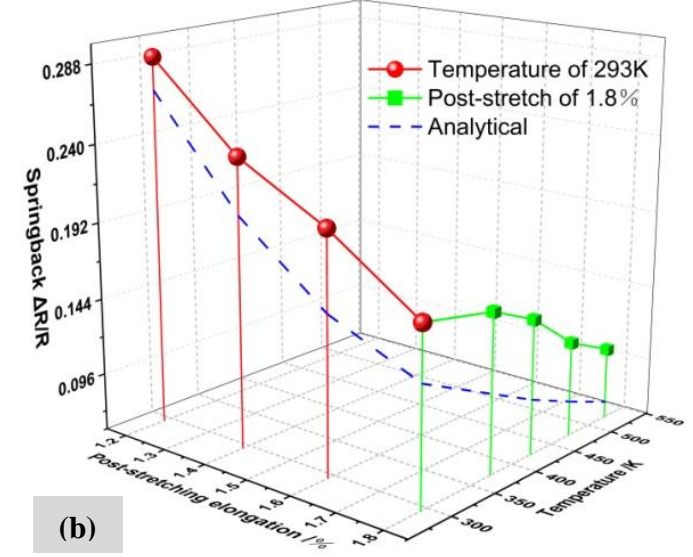

Figure 5. Stretch bending results (a)stretch bending parts (b)comparison of the experimental and analytical springback

\section{Conclusions}

The true stress-strain relationships obtained from the uniaxial tensile tests of 6063 aluminum alloy show that the flow stress increases with the decreases of test temperature at a given tensile velocity, but barely changes as the tensile velocity increases at the same temperature. The maximum elongation is achieved at $473 \mathrm{~K}$.

The springback of the profiles decreases with the increases of post-stretch elongation. The deformation behavior of material influenced by temperature has a great impact on the sprinigback. The forming accuracy of the aluminum profile can be improved in electrically assisted stretch bending process.

\section{References}

1. E. Corona, Int. J. Mech. Sci. 46, 433-448 (2004)

2. A.E. Tekkaya, N.B. Khalifa, G. Grzancic, Procedia Engineering (ELSEVIER, Nagoya, 2014)

3. F. Vollertsen, A. Sprenger, J. Kraus, J. Mater. Process. Tech. 87, 1-27 (1999)

4. G. Palumbo, L. Tricarico, J. Mater. Process. Tech. 184, 115-123 (2007)

5. D. Li, A. Ghosh, Mat. Sci. Eng. A 352, 279-286 (2003)

6. D. Li, A. Ghosh, J. Mater. Process. Tech. 145, 281293 (2004)

7. P.J. Bolt, N.A.P.M. Lamboo, P.J.C.M. Rozier, J. Mater. Process. Tech. 115, 118-121 (2001) 
8. S. Polak, P. Kaczyński, Z. Gronostajski, K. Jaskiewicz, Procedia Engineering (ELSEVIER, Cambridge, 2017)

9. H.D. Nguyen-Tran, H.S. Oh, S.T. Hong, IJPEM-GT, 2, 365-376 (2015) 